\title{
AÇÕES EM SAÚDE MENTAL ÀS FAMÍLIAS NOS DIFERENTES CONT EXTOS DE TRABALHO: revisão integrativa
}

\author{
M arinês AIRE Sa, Cristine M oraes ROOS ${ }^{b}$, A na Valéria F urquim G ON ÇALVESc, \\ Jacó Fernando SCHNEIDER ${ }^{\text {}}$, Agnes OL SCHOW SKY ${ }^{\mathrm{e}}$
}

\section{RESUM 0}

T rata-se de uma revisão integ rativa da literatura que objetivou identificar e analisar os estudos nos periódicos de enfermagem que focalizam as ações em Saúde M ental direcionadas às famílias. Para a coleta dos dados foram utilizados os descritores saúde mental, família e enfer magem, nas bases de dados da L iteratura L atino-A mericano e do Caribe em Ciências da Saúde (LILACS) e Scientific E lectronic L ibrary O nline (SciE L O). Os critérios de inclusão foram: artigos originais publicados em português, espanhol e inglês nos periódicos na área da enfer magem entre os anos de 2003 a 2008. Este trabal ho evidenciou uma predominância de estudos enfocando questões referentes à equipe de saúde e/ ou profissionais. Os achados indicam necessidade em produzir pesquisas voltadas para os usuários e familiares, o que pode contribuir para as ações em saúde mental da equipenos cenários da atenção psicossocial e apontar caminhos na direção de novas pesquisas.

D escritores: Saúde mental. F amília. Enfermagem psiquiátrica.

\section{RESUMEN}

E sta es una revisión integradora que tuvo como objetivo identificar y analizar los estudios en las revistas de enfermería que se centran en acciones de salud mental dirigidos a las familias en contextos laborales distintos. Para recopilar los datos, se utilizaron los descriptores dela salud mental, la familia y de enfermería en las bases de datos de A mérica $L$ atina y del Caribe de Ciencias de la Salud (L IL ACS) y Scientific E lectronic L ibrary Online (S ciE L O). L os criterios de inclusión fueron: artículos originales publicados en portugués, español el nglés en las revistas en el campo dela enfermería, entre los años 2003 y 2008. E ste trabajo mostró un predominio de los estudios centrados en las cuestiones de personal de salud y/ o profesional, indicando la necesidad de producir investigación de los usuarios y las familias. Se creeque estos hallaz gos pueden ser degran valor para los equipos en los escenarios de la atención psicosocial y señalar el camino hacia la investigación.

Descriptores: Salud mental. Familia. E nfermería psiquiátrica.

Título: A cción en salud mental a familias en diferentes contextos de trabajo: revisión integradora.

\section{ABST RACT}

This is an integrative review aimed to identify and analyze studies in nursing journals that focus on mental health actions directed to families in differ ent work contexts. To collect data, we used the descriptors mental health, family and nursing in the databases of the L atin American and Caribbean in H ealth Sciences (LIL ACS) and Scientific E lectronic Library Online (S CiE L O). Inclusion criteria were: original articles published in Portuguese, Spanish and E nglish in journals in the field of nursing between the years 2003 to 2008. T his work show ed a predominance of studies focusing on issues of health staff and/ or professional, indicating a need to produce research for users and families. It is believed that these findings may be of great value to the teams in the scenarios of psychosocial care and point the way tow ard further research.

D escriptors: M ental health. Family. P sychiatric nursing.

Title: Action in mental health to families in different contexts of work: integrative review.

\footnotetext{
a M estre em Enfermagem, Professora do Curso de G raduação em Enfermagem da U niversidade Regional Integrada do A Ito U ruguai e das M issões (U RI), Campus de F rederico Westphalen, Rio Grande do Sul, Brasil.

b Enfermeira E specialista em Saúde M ental Coletiva, M estranda em Enfermagem pelo Programa de Pós-G raduação em Enfermagem da U niversidade Federal do Rio Grande do Sul (PPGENF/ U FRGS), Porto Alegre, Rio Grande do Sul, Brasil.

c M estranda em Enfermagem pelo PPGENF/UFRGS, Enfermeira do Hospital de Clínicas de Porto Alegre (HCPA), Porto Alegre, Rio Grande do Sul, Brasil.

d D outor em Enfermagem, Professor do PPGENF/UFRGS, Porto A legre, Rio Grande do Sul, Brasil.

e D outora em Enfermagem Psiquiátrica, Professora do PPGENF/UFRGS, Porto Alegre, Rio G rande do Sul, Brasil.
} 


\section{INT RODUÇÃO}

No contexto que antecede o movimento da Reforma Sanitária, o sujeito em sofrimento psíquico não tinha voz ativa, sua história era marcada pela segregação e a responsabilidade pelo seu cuidado era atribuída à instituição hospitalocêntrica. Nesse cenário, as famílias renunciavam a qualquer participação no tratamento, assumindo a tarefa de identificar a desorganização mental, encaminhar o familiar, visitá-lo e fornecer informações sobre o paciente para equipe de saúde A família era vista como um sistema doente, como a causa do adoecimento, ou seja, transferia-se a ela a culpabilização pela doença ${ }^{(1,2)}$.

O M ovimento da Reforma Psiquiátrica preconiza a mudança do paradigma da psiquiatria clássica, questiona o modelo manicomial e propõe novos dispositivos de atendimento e tratamento, rompendo com os paradigmas do modelo asilar, preconizando o respeito às diferenças e à subjetividade dos indivíduos/ famílias, atribuindo um novo lugar social para a loucura e, para isso, instaurando um M odelo Psicossocial de Cuidado ${ }^{(3-5)}$. Para que tais mudanças sejam efetivas é necessário que elas transcendam a reestruturação física dos espaços, fundamentado no deslocamento da assistência psiquiátrica hospitalocêntrica para serviços territorializados. Além disso, esse modelo propõe que fatores políticos, biopsíquicos e sócio-culturais sejam tomados como determinantes das doenças( ${ }^{(4)}$.

D esse modo, a família assume o papel de protagonista no projeto terapêutico dos sujeitos com sofrimento psíquico. E ntretanto, a historicidade baseada no estigma, segregação, tutela e no modelo hegemônico biomédico ainda influenciam as ações em Saúde M ental nos diversos cenários de trabaIho. Se por um lado, os discursos acadêmicos fundamentados nos pressupostos da Reforma Psiquiátrica e Política de Saúde M ental ${ }^{(6)}$ priorizam a inserção da família no projeto terapêutico, por outro, os profissionais, os serviços de saúde e a sociedade, muitas vezes, consideram a família da mesma forma de outrora.

Diante desse cenário, a utilização de tecnologias leves, como o vínculo e o acolhimento são dispositivos potenciais para a integralidade da atenção ao usuário e família(7). A ssim, a Estratégia de Saúde da F amília (ESF), vem ao encontro dos pressupostos da Reforma Psiquiátrica, uma vez que desempenha um papel de articuladora da rede de Saúde M ental, pautada no território e na construção de uma rede ampliada de serviços de saúde. A lém disso, a ESF visa à superação do model o hospitalocêntrico e reorganização da produção de cuidados propondo uma atenção centrada na família, entendida e percebida a partir de seu ambiente físico e social e não somente focada indivíduo doen$\mathrm{te}^{(5,8)}$.

N esse espaço de cuidado às famílias, o acoIhimento constitui-se em uma tecnologia leve das ações em saúde, priorizando a atenção ao usuário por meio da escuta qualificada, da valorização das queixas e da identificação de suas necessidades, al iando a resolubilidade, a integralidade na assistência e a qualidade do serviço prestado ao usuário/ família(9).

Diante do panorama exposto, questiona-se: o novo modelo de Atenção Psicossocial atende os pressupostos da Reforma Psiquiátrica e inclui a família no processo de cuidado? Os profissionais consideram as famílias como usuárias dos serviços, uma vez que elas necessitam de atenção e apoio formal para manter sua própria saúde no processo de tratamento do seu familiar em sofrimento psíquico?

N esse sentido, o desafio que está posto é considerar a "loucura" como um fenômeno coletivo e social, delineando uma nova postura em relação ao sujeito que sofre psiquicamente, visando a um novo posicionamento do sujeito acerca de sua condição de "doente passivo sofredor" para uma atitude de "auto-administração", reconhecendo-se como agente que possui em si as possibilidades de modificar o contexto vivido.

Com base nessas considerações o presente estudo teve como objetivo identificar e analisar os estudos que focalizam as ações em Saúde M ental direcionadas às famílias nos diferentes contextos de trabal ho, publicados em periódicos de enfermagem.

\section{MÉTODOS}

A revisão integrativa é um método amplo que permite incluir estudos com diferentes abordagens metodológicas, agrupando resultados obtidos de um conjunto de pesquisas primárias com temática idênticas ou similares ${ }^{(10,11)}$. 0 seu objetivo é sintetizar e analisar esses dados para desenvolver uma explicação mais abrangente de um fenômeno es- 
pecífico a partir da síntese ou análise dos achados dos estudos, com propósitos teóricos e/ ou intervencionistas ${ }^{(12,13)}$.

As etapas que conduziram esta revisão integrativa sobre as ações em Saúde M ental às famílias no diferentes contextos de trabalho foram: formulação do problema; coleta de dados; avaliação dos dados; análise e interpretação dos dados; apresentação dos resultados e conclusões ${ }^{(12)}$.

A formulação do problema contemplou a elaboração da questão norteadora deste estudo: qual a produção do conhecimento da enfermagem em Saúde $M$ ental com enfoque nas ações direcionadas aos usuários e famílias nos diferentes contextos de trabalho?

A coleta de dados foi realizada por duas pesquisadoras, de forma independente, em julho de 2009, nas bases de dados da Literatura LatinoA mericano e do Caribe em Ciências da Saúde (LILACS) e Scientific Electronic Library Online (SciE LO ), utilizando-se os Descritores em Ciências da Saúde (D eCS): Saúde M ental, F amília eE nfermagem.

Os critérios de inclusão foram: artigos originais publicados em periódicos na área da enfermagem, de acordo com a classificação pelo $Q$ ual is de extrato $A$, que inclui $A 1$ eA 2 , e $B$ incluindo $B 1$, B2 e B3 da Coordenação de A perfeiçoamento de Pessoal em Nível Superior (CAPES), entre os anos de 2003 a 2008, disponíveis on-line e publicados em português, espanhol ou inglês.

0 levantamento bibliográfico no LILACS e SCiE L O totalizou, respectivamente, 49 e 25 publicações. A primeira seleção dos trabalhos foi feita a partir da leitura dos títulos e resumos de todos os artigos identificados na busca eletrônica, os quais se relacionavam com 0 objetivo da pesquisa. N essa etapa, foram selecionados 20 artigos no LILACS e 14 no SciE LO O. Posteriormente, realizou-se a busca e leitura dos estudos na íntegra.

Entre as publicações encontradas, seis fizeram parte de mais de uma base de dados e para a inclusão foi considerado 0 artigo encontrado na base de dados de maior produção. Foram excluídos quatro estudos bibliográficos, duas publicações de reflexão e outra por não se enquadrar nono foco de estudo em questão.

Assim, a amostra final foi constituída por 22 estudos. Para a avaliação dos dados elaborou-se um instrumento para a coleta das informações visando responder a questão norteadora desta revisão.
A análise e interpretação dos dados foram realizadas de forma organizada e sintetizada por meio da elaboração de um quadro sinóptico que compreendeu os seguintes itens: identificação do estudo; objetivos, ano e periódico de publicação; Qualis do periódico; delineamento do estudo; temática; referencial teórico-metodológico; sujeitos da pesquisa; e, principais resultados.

Os artigos selecionados foram analisados na integra e agrupados por áreas temáticas, A análise quantitativa ocorreu por meio da análise estatística de frequência absoluta e relativa. T ambém realizou-se uma comparação dos achados nos artigos relacionados ao desenvolvimento das ações em Saúde M ental pelos profissionais direcionados às famílias.

Quanto aos aspectos éticos, salienta-se que os preceitos de autoria e referenciamento das obras consultadas foram respeitados. Como o estudo configura-se como uma revisão integrativa da produção existente e disponível sobre a temática, entendeu-se aceitável dispensar sua submissão a um Comitê de Ética em Pesquisa.

\section{RESULTADOS E DISCUSSÃO}

$\mathrm{Na}$ T abela 1, apresenta-se a distribuição das publicações de enfermagem na atenção à Saúde $M$ ental às famílias nos periódicos de enfer magem no período de 2003 a 2008.

T abela 1 - Distribuição das publicações de enfermagem na atenção à Saúde $M$ ental às famílias nos periódicos de enfermagem no período de 2003 a 2008. Porto Alegre, RS, 2009.

\begin{tabular}{lcr}
\hline Periódico & $\mathbf{n}$ & $\%$ \\
\hline Revista Latino-A mericana de Enfermagem & 2 & 9,0 \\
Revista Eletrônica de Enfer magem & 1 & 4,5 \\
Revista de E nfer magem da U niversidade & & \\
do E stado do Rio de Janeiro (U E RJ) & 3 & 13,6 \\
Escola A nna N ery Revista de Enfermagem & 3 & 13,6 \\
Cogitare Enfer magem & 5 & 22,7 \\
Revista Brasileira de Enfer magem & 3 & 13,6 \\
Revista da E scola de Enfermagem da & & \\
Universidade de São Paulo (U SP) & 3 & 13,6 \\
Revista G aúcha de Enfermagem & 1 & 4,5 \\
Texto \& Contexto: Enfermagem & 1 & 4,5 \\
\hline Total & $\mathbf{2 2}$ & $\mathbf{1 0 0 , 0}$ \\
\hline
\end{tabular}


Dos 22 artigos analisados, verificou-se que o maior número de publicações concentra-se no ano de 2006 (oito) e 2005 (cinco) nos anos de 2004 (três), 2008 (três) e no ano de 2007 (dois). Cabe salientar que os estudos referentes ao ano 2003 não estavam disponíveis on-line na integra. 0 periódico Cogitare Enfermagem do Departamento de Enfer magem da U niversidade Federal do Paraná, classificado pelo sistema Qualis/CAPES como B3, apresentou o maior percentual de publicações $(22,7 \%)$.

A Tabela 2 apresenta as publicações de enfermagem na atenção à Saúde M ental às famílias de acordo com o Referencial Teórico M etodológico utilizado pelos autores.

T abela 2 - Distribuição das publicações de enfermagem na atenção à saúde mental às $F$ amílias nos periódicos de enfermagem no período de acordo com o Referencial Teórico M etodológico de 2003 a 2008. Porto Alegre, RS, 2009.

\begin{tabular}{lcr}
\hline Referencial T eórico-M etodológico & $\mathbf{n}$ & $\%$ \\
\hline M étodo de história oral temática & 1 & 4,5 \\
M aterial ismo H istórico e Dialético & 3 & 13,6 \\
Referencial das representações sociais na & \\
perspectiva dos pressupostos de M oscovici & 3 & 13,6 \\
A bordagem sistêmica: M odelo Cal gary de & & \\
Avaliação da Família e M odel o Calgary de & & \\
Inter ven ção na família & 1 & 4,5 \\
Fenomenologia & 1 & 4,5 \\
Processo Clinical Caritas de Jean Watson e & & \\
Processo de Cuidar de Lacerda & 1 & 4,5 \\
Teoria Ego-E cológica de M arisa Zavalloni & 1 & 4,5 \\
Etnometodologia & 1 & 4,5 \\
Construcionismo Social & 1 & 4,5 \\
Não especificado & 8 & 36,4 \\
\hline Total & $\mathbf{2 2}$ & $\mathbf{1 0 0 , 0}$ \\
\hline
\end{tabular}

A partir dessa constata-se a que $36,4 \%$ dos estudos analisados não especificaram a utilização de Referencial Teórico-M etodológico. No achados, $27,2 \%$ das pesquisas indicam uma tendência de apropriação de quadro teórico das ciências humanas, sociais e comportamentais como, por exemplo, o Referencial T eórico-M etodológico do $M$ aterialismo Histórico e Dialético e Referencial das Representações Sociais com base nos pressupostos de M oscovici. Isto pode ser justificado pela necessidade de compreensão dos aspectos subjetivos da experiência prática vivenciada pela enfermagem, acarretando subsídios às ações no cotidiano dos serviços de Saúde M ental.

A T abela 3 apresenta a distribuição das publicações de E nfermagem na Atenção à Saúde M ental segundo tipo do estudo.

T abela 3 - Distribuição das publicações de enfer magem na atenção à saúde mental às F amílias nos periódicos de enfermagem no período de 2003 a 2008 segundo tipo do estudo. Porto Alegre, RS, 2009.

\begin{tabular}{lrr}
\hline T ipo de estudo & n & \multicolumn{1}{c}{$\%$} \\
\hline Pesquisa Qualitativa & 20 & 91,0 \\
Pesquisa Quanti-quali & 1 & 4,5 \\
Pesquisa Quantitativa & 1 & 4,5 \\
\hline Total & $\mathbf{2 2}$ & $\mathbf{1 0 0 , 0}$ \\
\hline
\end{tabular}

Conforme os dados da T abela 3, o maior percentual de publicações de enfermagem em Saúde M ental (91\%) está classificado como artigo de pesquisa qualitativa. Entre os quais, cinco classificamse com estudo de caso. A pesquisa com abordagem qual itativa do tipo estudo de caso, visa a investigar os fenômenos inseridos no contexto real de maneira profunda e intensa, deste modo, possibilita ao pesquisador uma maior compreensão sobre os fenômenos individuais, sociais, pol íticos e degrupo ${ }^{(14)}$.

A pesquisa qualitativa possibilita uma meIhor compreensão dos fenômenos e uma maior aproximação com a realidade dos sujeitos, no caso as famílias. U ma vez que o foco é estudar o universo de significados, motivos, aspirações, crenças, valores e atitudes, o que corresponde a um espaço mais profundo das relações, dos processos e dos fenômenos que não podem ser reduzidos ou mensuráveis somente através da operacionalização de variáveis ${ }^{(15)}$.

A T abela 4 apresenta a distribuição das publicações de E nfermagem na A tenção à Saúde M ental de acordo com os sujeitos da pesquisa.

D urante a anál ise dos artigos, buscou-se identificar os sujeitos em estudo com o propósito de visualizar a abrangência dos pesquisadores da área em Saúde M ental no que se refere à integração dos diversos atores envolvidos no processo de cuidado, ou seja, família, profissionais e usuários. Denota-se a predominância dos estudos tendo como 
sujeitos das pesquisas os profissionais de saúde $(40,5 \%)$. Esses estudos focalizam o cuidado em Saúde M ental da equipe no contexto da ESF, além disso, considerou-se que o foco do trabalho neste cenário é a família. Vale ressaltar o aumento das produções envolvendo os diversos atores, o que se constitui em um campo amplo ainda com lacunas a serem exploradas.

T abela 4 - Distribuição das publicações de enfermagem na atenção à saúde mental às F amílias nos periódicos de enfermagem no período de 2003 a 2008 segundo sujeitos do estudo. Porto A legre, RS, 2009.

\begin{tabular}{llr}
\hline Sujeitos da Pesquisa & n & $\%$ \\
\hline Profissionais de saúde & 9 & 40,0 \\
Família & 3 & 13,6 \\
Família e usuários & 3 & 13,6 \\
U suários & 3 & 13,6 \\
Profissionais de saúde, usuários e familiares & 2 & 9,0 \\
U suários e Profissionais de saúde & 1 & 4,5 \\
Cuidadores de pacientes psiquiátricos & 1 & 4,5 \\
\hline
\end{tabular}

T otal

22100,0

A T abela 5 a apresenta a distribuição das publicações de Enfermagem em Saúde M ental segundo área temática.

T abela 5 - D istribuição das publicações de enfermagem na atenção à Saúde $M$ ental às F amílias nos periódicos de enfermagem no período de 2003 a 2008 segundo a área temática. Porto Alegre, RS, 2009.

\begin{tabular}{lrr}
\hline T emática & $\mathbf{n}$ & \% \\
\hline Saúde M ental na Atenção Básica & 14 & 64,0 \\
Centro de A tenção Psicossocial (CA PS) & 7 & 32,0 \\
H ospital-dia & 1 & 4,0 \\
\hline T otal & $\mathbf{2 2}$ & $\mathbf{1 0 0 , 0}$ \\
\hline
\end{tabular}

Com base na T abela 5, evidencia-se a prevalência de estudos relacionados à Saúde M ental na A tenção Básica, dentre os quais, a maioria buscam analisar e identificar como são desenvolvidas as ações em Saúde M ental no contexto da E stratégia Saúde da F amília. D e acordo com os estudos o trabalho em Saúde M ental, nesse cenário, encontra- se em processo de transformação no sentido da inclusão social e da integralidade da assistência. No entanto, ainda carece de articulação com as condições sociais, econômicas e culturais e de profissionais comprometidos. A pesar dos avanços, a maioria as ações em Saúde M ental ainda são orientadas pela prática da psiquiatria tradicional, em que a medicalização e o encaminhamento são os instrumentos de escolha ${ }^{(16-23)}$. N essa perspectiva, a ESF pode ser vista como mediadora no processo de mudança das práticas no campo da Saúde M ental, articuladora da rede de Saúde M ental, bem como um elo para o fortalecimento e efetivação dos princípios do Sistema Ú nico de Saúde (SU S).

Entre as pesquisas analisadas, 27\% focalizam a família, o Centro de Atenção Psicossocial (CAPS) e os profissionais. Considera-se que esses atores desempenham papel relevante para a consolidação da Reforma Psiquiátrica, porém é necessário a articulação com as demais áreas para a construção de uma rede de cuidado em Saúde M ental, sendo o vínculo um dispositivo fundamental e estratégico para tal articulação(24-33). U m aspecto relevante observado pelos autores e que vem reforçar a estratégia de atenção psicossocial, foi a angústia e tristeza dos usuários diante da inter nação no hospital psiquiátrico, em função da maneira como é prestado o cuidado em instituição de internação ${ }^{(31)}$. T ambém identificou-se em alguns estudos a importância da atuação da equipe enquanto integradora desta parceria ${ }^{(27,31,32)}$.

Para tanto, 0 ambiente deve ser acolhedor, os profissionais devem proporcionar um cuidado singular que respeite a subjetividade de cada indivíduo e de cada família, compreendendo suas limitações e incentivando suas habilidades, estimulando o protagonismo e promovendo a autonomia da família( ${ }^{(34)}$. Contribuindo com essa discussão, 0 cuidado transpessoal propicia a transformação da relação de poder entre profissionais, usuários e família, criando uma oportunidade para promoção da integralidade na assistência(35).

D esse modo, a valorização do familiar, o respeito e a escuta qualificada são imprescindíveis para que a atenção psicossocial realmente possa ocorrer e possamos pensar em projetos de vida ${ }^{\left({ }^{30}\right)}$. O utro aspecto rel evante diz respeito ao acolhimento da fala do familiar como elemento importante do cuidado das equipes no setting terapêutico. Essa prática precisaria ser inserida ativamente, refletindo uma exigência institucional(29). 
Dois estudos destacaram a importância dos grupos como um fator que colabora na inserção da família e possibilita a promoção em saúde mental por meio de ações de educação em saúde ${ }^{(32,36)}$. A atividade de natureza grupal exerce influências benéficas no processo de reabilitação psicossocial, pois cada participante é diferente e ao mesmo tempo possui necessidades semelhantes para realizar uma tarefa ${ }^{(37)}$. Pesquisa realizada com familiares cuidadores de "doente esquizofrênico" indicou que qualidade de vida é ter saúde, poder trabalhar e sustentar a família, além disso, fatores como deixar de ir a igreja, não sair com amigos, deixar de trabalhar e não ter mais tempo para si mesmo interferem na vida destes cuidadores ${ }^{(38)}$. Em um dos estudos, ressal tou-se que o portador de sofrimento psíquico deveria ter a possibilidade de interagir com/ no contexto social em que vive, ou seja, que seu papel de cidadão transcenda os "muros" da instituição a qual está vinculado(29).

Com relação ao respeito pelas características individuais do sujeito em sofrimento psíquico, um dos estudos considera que algumas famílias constroem um olhar estigmatizante e segregador ${ }^{(39)}$. T ais concepções seriam geradoras de tristeza, angústia e medo e poderiam ser resquícios de uma atenção proveniente da psiquiatria clássica, caracterizada pelas práticas excludentes e manicomiais, que ainda perpassam o cotidiano social. A desconstrução do saber manicomial é um elemento importante para o resgate de cidadania/ identidade do indivíduo(28).

E mbora os autores centrem suas discussões na atenção por parte da enfer magem, salienta-se que, no cuidado em Saúde M ental, é necessária a participação dos familiares, e profissionais de outras áreas para a efetivação da Reforma Psiquiátrica dado que emergiu em um dos estudos, no qual os autores salientam que o cuidado e a responsabilização sejam compartilhados entre equipes e familiares comprometida com as necessidades dos usuários(32).

\section{CONSIDERAÇÕES FINAIS}

Por meio desta revisão integrativa, buscouse identificar e analisar os estudos publicados em periódicos de enfermagem que focalizam as ações em Saúde M ental direcionadas às famílias nos diferentes contextos de trabalho. Entre os principais resultados, destaca-se a predominância de pesqui- sas qualitativas, com utilização do Referencial Teórico do M aterialismo Histórico Dialético e das Representações Sociais de M oscovici, tendo como enfoque a Saúde M ental na A tenção Básica, predominantemente na ESF. Este trabalho evidenciou a ênfase dos estudos enfocando questões referentes à equipe de saúde e/ ou profissionais, no entanto observou-se que os estudos estão começando a incluir os demais atores envolvidos nesse processo.

Os achados dos estudos com enfoque na Atenção Básica sugerem a necessidade de maior preparo dos profissionais, tendo em vista a importância da E SF e da reforma psiquiátrica, enfatizando a real ização de um trabal ho conjunto, entre os familiares e as equipes de saúde da família. constatouse que ações de Saúde M ental desenvolvidas no contexto da ESF são orientadas pela prática da psiquiatria tradicional, onde a medicalização e o encaminhamento são os instrumentos de escolha.

$\mathrm{N}$ os estudos realizados nos CAPS, 0 acolhimento apresentou-se como um organizador do trabalho, o que vem a contribuir para mudança do modelo assistencial centrado na doença para outro, que considera a subjetividade do ser humano cuidado. Além disso, possibilita a inversão da assistência centrada nas tecnologias leves duras e duras para uma assistência centrada nas tecnologias leves e leves duras.

No estudo realizado com familiares e usuários em um hospital-dia, no qual a família foi representada, predominantemente, como fonte de afeto e apoio. 0 hospital-dia foi caracterizado como um local de tratamento e acolhimento, tanto familiares como usuários demonstraram acreditar nas possibilidades de progresso advindas do atendimento nesse tipo de serviço. Assim, identificou-se a Saúde M ental, nesse cenário de trabaIho, vinculada à recuper ação e ao tratamento.

Por meio desta revisão integrativa constatou-se que ainda há muito por fazer rumo à efetivação da Reforma Psiquiátrica. Assim, vislumbrase como grande desafio a construção de um "novo ol har", um "novo assistir" e repensar as ações em Saúde M ental por meio do uso de tecnologias leves como dispositivos para a produção do cuidado ao usuário e família quando estes se apresentam diante dos serviços de saúde em busca atendimento, seja ele, na atenção básica ou CAPS.

As ações em Saúde M ental devem ser desenvolvidas por profissionais comprometidos e 
permeadas de valores humanísticos, como alteridade, respeito, escuta qualificada, comprometimento e responsabilização com as necessidades relatadas pelo usuário durante o encontro profissional/ usuário/ família. Seguindo essa linha de pensamento, a assistência às famílias, torna-se um grande desafio da prática em Saúde M ental, exigindo dos profissionais estudos e aproximação desses atores sociais. Tendo em vista a importante e desafiadora tarefa de promoção do protagonismo do usuário/ família que se coloca aos profissionais que atuam na atenção em Saúde M ental, a atitude é inovar, planejar e implementar ações em Saúde M ental com este enfoque psicossocial. $\mathrm{N}$ esse sentido, é preciso, ainda, construir um novo paradigma e, conseqüentemente, uma nova linguagem, que possa dar conta da complexidade que envolve essas questões e propor a formação de redes de suporte de cuidado em saúde mental, em que haja efetiva participação da família.

Destaca-se a necessidade de ampliar as investigações voltadas a essa temática contribuindo com o exercício da enfermagem enquanto ciência e prática profissional.

\section{REFERÊ NCIAS}

1 W etzel C, Kantorski L. Reinaldo A. A inserção da família na assistência em Saúde M ental. Saúde D ebate. 2005;29(1):5-16.

2 Borba LO, Schwartz E, Kantorski LP. A sobrecarga da família que convive com a realidade do transtorno mental. Acta Paul Enferm. 2008;21(4):588-94.

3 A marante $P$, organizador. Psiquiatria social e reforma psiquiátrica. Rio de Janeiro: F iocruz; 2002.

4 Costa-Rosa A. 0 modo psicossocial: um paradigma das práticas substitutivas ao modo asilar. In: A marante $P$, organizador. E nsaios: subjetividade, saúde mental, sociedade. Rio de Janeiro: F iocruz; 2000. p. 141-68.

5 Hirdes A. Reabilitação psicossocial: dimensões teórico-práticas do processo. E rechim: FAPE S; 2001.

6 M inistério da Saúde (BR), Secretaria de Atenção à Saúde. L egislação em saúde mental: 1990-2004. 5a ed. Brasília (DF); 2004.

7 Franco T B, M erhy E E. PSF : contradições de um programa destinado à mudança do modelo assistencial. In: M ehy EE, M agal hães J r. H M, Rimoli J, Franco
T B, Bueno W S, organizadores. 0 trabal ho em saúde: ol hando e experienciando o SUS no cotidiano. São Paulo: Hucitec; 2003. p. 55-124.

$8 \mathrm{~N}$ unes $\mathrm{M}$, Juca VJ, Valentim CPB. A ções de saúde mental no Programa Saúde da Família: confluências e dissonâncias das práticas com os princípios das reformas psiquiátrica e sanitária. Cad Saúde Pública. 2007;23(10):2375-84.

9 Ramos DD, Lima M ADS. A cesso e acolhimento aos usuários em uma unidade de saúde de Porto Alegre. Cad Saúde Pública. 2003;19(1):27-34.

10 Pompeo DA, Rossi LA, G alvao CM . Revisão integrativa: etapa inicial do processo de validação de diagnóstico de enfermagem. A cta Paul E nferm. 2009;22 (4):434-8.

11 W hittemore $R$, Knafl $K$. T he integrative review: updated methodology. J Adv N urs. 2005;52(5): 546-53.

12 Cooper HM. The integrative research review: a systematic approach. N ewbury Park: Sage; 1989.

13 Crossetti M G 0, Antunes M, Anziliero F. Revisão integrativa da literatura: referenciais metodológicos de pesquisa na enfer magem. I n: A nais do 150 Seminário N acional de Pesquisa em Enfer magem; 2009 jun 08-11; Rio de Janeiro, Brasil. Rio de Janeiro: $A B E n / R J ;$ 2009. p. 1618-20.

14 Y in RK. Estudo de caso: planejamento e métodos. 3a ed. Porto A legre: Bookman; 2005.

15 M inayo M CS. 0 desafio do conhecimento: pesquisa qualitativa em saúde. 10ª ed. São Paul o: H ucitec; 2007.

16 Buchele F, L aurindo D LP, Vanessa F B, Elza BSC. A interface da saúde mental na A tenção Básica. Cogitare Enferm. 2006;11(3):226-33.

17 Koga M , F uregato AR, Santos JLF. O pinions of the staff and users about the quality of the mental health care delivered at a family health prog ram. Rev $L$ atino-Am E nfer magem. 2006;14(2):163-9.

18 Souza AJF, M atias GN, Gomes KF, Parente ACM . A saúde mental no Programa de Saúde da Família. Rev Bras Enferm. 2007;60(4):391-5.

19 Silva AT M C, Silva CC, Ferreira F ilha M O, Nóbrega $M M L, B a r r o s ~ S$, Santos KKG. A saúde mental no PSF e o trabalho de enfermagem. Rev Bras Enferm. 2005;58(4):411-5. 
20 N ascimento AM B, Violante AB. A tenção em saúde mental: a prática do enfermeiro e do médico do Programa Saúde da Família de Caucaia-CE . Cogitare E nferm. 2004;9(1):84-93.

21 Sousa KB, Ferreira Filha M 0, Silva AT M C. A práxis do enfer meiro no Programa Saúde da Família na A tenção à Saúde M ental. Cogitare E nferm. 2004;9 (2):14-22.

22 Pereira M AO, Barbieri L, Paula VP, Franco M SP. Saúde mental no Programa de Saúde da F amília: conceitos dos agentes comunitários sobre o transtorno mental. Rev Esc Enferm USP. 2007;41(4):56772.

23 Souza RC, Scatena M CM . Produção de sentidos acerca da família que convive com o doente mental. Rev L atino-A m E nfermagem. 2005;13(2):173-9.

24 M oura LS, Kantorski LP, Galera SAF. Avaliação e intervenção nas famílias assistidas pela equipe de saúde da família. Rev G aúcha E nferm. 2006;27 (1):35-44.

25 Oliveira R M P, Loyola CM D. Pintando novos caminhos: a visita domiciliar em saúde mental como dispositivo de cuidado de enfermagem. E sc A nna N ery Rev Enferm. 2006;10(4):645-51.

26 G uimarães F G, Ferreira Filha M O. Repercussões da terapia comunitária no cotidiano de seus participantes. Rev Eletrônica Enferm. 2006;8(3):40414.

27 Jorge M SB, Ramirez ARA, Lopes CHAF, Queiroz M VO, Bastos VB. Representações sociais das famílias e dos usuários sobre participação de pessoas com transtorno mental. Rev Esc Enferm U SP. 2008;42 (1):135-42.

28 Coimbra VCC, Kantorski LP. 0 acolhimento em Centro de Atenção Psicossocial. Rev Enferm UERJ. 2005; 13(1):57-62.

\section{Endereço da autora / Dirección del autor / Author's address:}

$M$ arinês Aires

Rua Comércio, 724, Centro

98400-000, Frederico W estphalen, RS

E - mail: marynesayres@yahoo.com.br
29 Hirdes A, Kantorski LP. A família como um recurso central no processo de reabilitação psicossocial. Rev Enferm UERJ. 2005;13(2):160-6.

30 M ello M , F uregato AR. Representações de usuários, familiares e profissionais acerca de um centro de atenção psicossocial. E sc A nna N ery Rev E nferm. 2008; 2(3):457-64.

31 Oliveira AM N, L unardi VL, Santos M R. Repensando o modo de cuidar do ser portador de doença mental e sua família a partir de Heidegger. Cogitare E nferm. 2005;10(1):9-15.

32 Schrank G, Olschowsky A. O Centro de Atenção Psicossocial e as estratégias para inserção da família. Rev E sc Enferm U SP. 2008;42(1):127-34.

33 F uregato AR, Silva E C. A doença mental vivida por um paciente psiquiátrico: suas percepções. E sc A nna Nery Rev E nferm. 2006;10(4):652-9.

34 Stuart GW, Laraia M T. Enfermagem psiquiátrica: princípios e prática. 6a ed. Porto Alegre: Artmed; 2001.

35 Fonseca AL M, Lacerda M R, M aftum M A. O cuidado transpessoal de enfermagem no domicílio ao portador de transtorno mental e sua família. Cogitare E nferm. 2006;11(1):7-15.

36 M acêdo VCD, M onteiro ARM . E ducação e Saúde M ental na família: experiência com grupos vivenciais. T exto Contexto Enferm. 2006;15(2):222-30.

37 Zimermann DE. F undamentos básicos das grupo terapias. $2^{a}$ ed. Porto Alegre: A rtmed; 2000.

38 T eixeira M B. Q ualidade de vida de familiares cuidadores do doente esquizofrênico. Rev Bras Enferm. 2005;58(2):171-5.

39 Colvero L A, I de CAC, Rolim M A . F amília e doença mental: a difícil convivência com a diferença. Rev E sc E nferm U SP. 2004;38(2):197-205.

Recebido em: 18/ 10/ 2009

A provado em: 02/ 06/ 2010 